\title{
The relationship between arousal level and habituation of the orienting reaction*
}

\author{
GUNILLA BOHLIN \\ Liniversity of Uppsala, Uppsala, Sweden
}

\begin{abstract}
Three groups of $10 \mathrm{Ss}$ each were subjected to a habituation procedure with an auditory stimulus. Recordings were made of EEG and skin conductance. One group was tested in the morning after one night of sleep deprivation (SD) and the two others in the morning and afternoon following an ordinary night of sleep. The SD group showed a faster occurrence of sleep than did the other two groups. whereas the groups did not differ in rate of habituation of skin conductance responses to stimuli. Still, correlations between measures of arousal and habituation over all Ss were significant.
\end{abstract}

The relationship between arousal level and habituation of the orienting reaction (OR) is an issue for theories of habituation (Lynn, 1966: see also Sokolov, 1963, and Groves \& Thompson, 1970). The problem involves two aspects that both have to be considered in an empirical analysis. A dearousing effect of prolonged habituation has been claimed (Sokolov, 1963) and, on the other hand, arousal level might affect rate of habituation (Sokolov, 1963: Lader \& Matthews, 1968). For the former aspect, empirical support has been presented (Bohlin, 1971), whereas for the latter, the empirical picture is less clear.

The findings differ somewhat for different components of OR, but even for GSR, the one most investigated, the results are inconclusive. An inverse relation between arousal level and rate of habituation has been reported by Scholander $(1960,1961)$, Johnson (1963), Koepke and Pribram (1966), Katkin and McCubbin (1969), Lader and Wing (1966), Thayer and Silber (1971), Maltzman, Smith, Kantor, and Mandell (1971), Bohlin (1972, 1973), whereas Tizard (1966) and McDonald, Johnson, and Hord (1964) conclude that there is no effect of arousal on rate of habituation. Finally, it has also been claimed (Johnson \& Lubin, 1967) that habituation is delayed at the low end of the arousal continuum, i.e., if an identification of sleep and low arousal is accepted. Although the evidence in favor of an inverse relation seems to be in the majority, its force is weakened by the fact that most of the studies have used individual differences rather than experimental manipulations to obtain variation in level of arousal. Moreover, some of the few studies using experimental manipulations can be criticized on the basis that their manipulations are likely to affect the habituation in other ways than through arousal. Thus, manipulations imparting signal value to the stimuli would be seen as dubious, as would pharmacological manipulations as long as the exact physiological bases of habituation and arousal cannot be related to the action of the specific drug. A second factor complicating an

*This study was supported by a grant from the Swedish Council for Social Science Research. interpretation of the above results is the difference in the conceptualization of rate of habituation. According to the two main views, the rate can be seen either as the time to reach a habituation criterion or the slope of the response function. Since initial level of responding affects these measures oppositely. similar results can be the basis of opposite conclusions, depending on which of the conceptualizations is employed (compare Tizard. 1966, and Maltzman et al, 1971: see also Bohlin. 1973, and Hinde, 1970).

In the present investigation. the intention was to bring about arousal differences by employing the diurnal variation (Colquhoun, 1971) and deprivation of sleep. The effect on habituation of the skin conductance component of $\mathrm{OR}$ was evaluated. taking the alternative ways to conceive of rate of habituation into account. To substantiate the effectiveness of the manipulation, the occurrence of sleep was ascertained. thus relating to the previously adopted notion of arousal decrease (Bohlin. 1971).

\section{METHOD}

Subjects

Thirty students at the Lniversity of Lppsala. 13 males and 17 females, were paid to serve as Ss.

\section{Apparatus}

The physiological recordings were made on a Hewlett-Packard two-channel polygraph. The EEG was recorded by a parieto-occipital channel using Hewlett-Packard electrodes and paste, and the skin conductance (SC) measures were recorded from Offner-Beckman $\mathrm{Ag}-\mathrm{AgCl}$ electrodes fastened at the second and third fingers of the left hand with Offner-Beckman electrode paste as contact medium. Skin conductance responses (SCRs) were obtained by a constant voltage circuit as a conductance rise against a stabilized baseline achieved by the help of a smoothing capacitor (Edelberg. 1967). while skin conductance level was read separately from the voltmeter. The paper speed was shifted between $1 \mathrm{~mm} / \mathrm{sec}$ and $5 \mathrm{~mm} / \mathrm{sec}$ in a way that each $2-\mathrm{min}$ period contained a $30-\mathrm{sec}$ epoch of the higher speed.

The tone stimuli were produced by an Oltronix RC oscillator. Type RCO-6K. The length of the intersignal intervals were automatically controlled by a tape recorder, whereas the signal length was controlled by an electronic decade timer. 


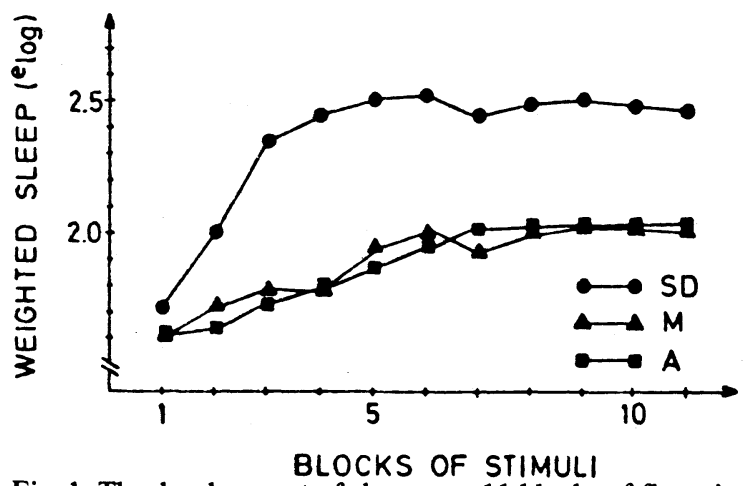

Fig. 1. The development of sleep over 11 blocks of five stimuli for the sleep-deprived (SD) group and for the groups tested in the morning (M) and in the afternoon (A).

Design and Procedure

The Ss were allocated to three different groups of 10 Ss each. For one of the groups. the Ss were individually subjected to one night of sleep deprivation (SD) before being exposed to the experimental procedure in the following morning. The other two groups both had an ordinary night of sleep before the day of the experiment, but the time of testing differed. For one of the groups, it occurred at 8 a.m. [the morning (M) group], while for the other one, it occurred around 2 p.m. [the afternoon (A) group].

The Ss of the SD group arrived at the institute at 10 p.m. and spent the night there together with an E. Some ratings were made once every hour, but otherwise no tasks were given during the night. At about 8 a.m., the $\mathrm{S}$ took a 5 -min walk from the institute to the laboratory. Thereafter, the procedure was identical for the three groups. Electrodes for physiological recordings were applied. and the $\mathrm{S}$ was seated in a soundproof cubicle in the laboratory. The instruction was given, telling the $\mathrm{S}$ that no overt responses were requested but that the $S$ was to sit with closed eyes for half an hour while physiological recordings were made. The instruction also included a passage trying to control the attitude towards falling asleep (see Bohlin, 1971). The habituation procedure started. containing 55 presentations of a 4 -sec. $1,000-\mathrm{Hz}, 80 \mathrm{~dB}$ tone, with an interstimulus interval of $20-40 \mathrm{sec}$

\section{Dependent Variables}

\section{EEG Measures}

The tracing from the $30-\mathrm{sec}$ epochs of high paper speed were scored as belonging to one of the following categories: (1) Alert-Low-voltage fast pattern (beta waves) and/or alpha waves; not more than $10 \%$ slow activity (delta). This category corresponds to the "awake" stage in Oswald's (1962) terminology. (2) Drowsy-Alpha activity with occurrence of delta activity (at least 10\%); corresponds to Oswald's Stage A. (3) Asleep-Disappearance of alpha (not more than 10\%) and appearance of a low-voltage pattern with slow waves (delta); Stages B. C. D, or E according to Oswald (1962).

To reduce the risk of a contamination between the activated alert and the asleep category, account was taken to the responses evoked by the stimuli. e.g., if alpha activity was provoked or not. If two successive epochs were found to belong to different categories. the tracing in between was examined for the point of change. In most cases, such a point could be identified. but where this was impossible, the change was considered to occur half way between the two epochs. In this way, a continuous scoring of the EEG was attempted.

Lsing the stimuli as time units, each stimulus was then scored as occurring during either of the three categories. and time to sleep onset was given by the number of stimuli before the first one belonging to the "asleep" category. For each successive block of five stimuli, a weighted sleep score was calculated by arbitrarily giving the weights 1,2 . and 3 to the stimuli belonging to the three categories and then obtaining the total weighted amount.

\section{Skin Conductance Measures}

A skin conductance response ( $\mathrm{SCR}$ ) was defined as a change of at least $.003 \mathrm{log} \mu$ mhos. For each block of five stimuli, the number of specific SCRs, occurring between 1 and $5 \mathrm{sec}$ following stimulus onset, was ascertained and their mean magnitude calculated. The time to habituation was scored as the number of stimuli to three successive zero responses.

The spontaneous SCRs were sampled during a 20 -sec interval before each tone, and the total number for each stimulus block was counted.

The skin conductance level (SCL), finally, was read three times in each block of stimuli. The readings were transformed to natural logarithm $\left({ }^{e} \log \right)$ units and an average score for each stimulus block was obtained.

\section{RESULTS}

Analyses of variance were carried out for the dependent variables, using groups or groups and blocks as factors. In the latter case, the block effect was also analyzed with respect to trend components (Kirk, 1968). Because of positively skewed distributions for weighted sleep and spontaneous SCRs, a log transformation was made before performance of the statistical test.

\section{EEG Variables}

The groups differed in time to sleep onset, $\mathrm{F}(2,27)=$ $8.81, \mathrm{p}<.01$, the means being: SD, 22.7; M, 48.0; $\mathrm{A}$, 45.5. Thus, the SD group had shorter time to sleep onset than either of the other two groups $[\mathrm{p}<.01$, according to Tukey's test (Kirk, 1968)] .

Figure 1 presents the development of sleep for the three groups. There was an overall difference between groups, $F(2,27)=8.18, p<.01$, and a significant effect of blocks, $F(10,270)=23.89, p<.001$, the latter comprising both linear, $F(1,270)=202.63, p<.001$, and quadratic, $F(1,270)=30.32, p<.001$, components. The Groups by Blocks interaction was also significant, $\mathrm{F}(20,270)=2.17, \mathrm{p}<.01$, signifying a difference in quadratic trends between the groups, $F(2,270)=10.69$, $\mathrm{p}<.001$. The results are interpreted to mean that the SD groups had a more rapid arousal decrease than the other two groups (see Fig. 1).

\section{Electrodermal Variables}

The means in time to habituation for the three groups were: SD, $6.3 ; \mathrm{A}, 7.7 ; \mathrm{M}, 6.5$; they did not yield a significant difference, $F(2,27)<1$.

Figure 2 illustrates the habituation of specific SCRs. For both probability and magnitude of response, an effect of blocks was seen, $F(10,270)=8.47, p<.001$, and $F(10.270)=3.59, p<.001$, respectively. In both 
cases. the development over blocks comprised linear, $F(1.270)=29.44 . p<.001$, and $F(1.270)=1.7$. $p<.05$. as well as quadratic. $F(1.270)=11.58$. $\mathrm{p}<.001$. and $\mathrm{F}(1.270)=14.51 . \mathrm{p}<.001$, components. In neither case was there a significant effect of groups or Groups by Blocks. Thus, for both measures, there was a curved overall decrease with no differences between groups.

The results for spontaneous $S C R s$ and $S C L$ are plotted in Fig. 3. There was a significant effect of blocks for both variables, $F(10.270)=2.78, p<.01$, for the former, and $F(10.270)=9.50, p<.001$, for the latter. The decrease for the SCL held both a linear. $F(1.270)=$ 89.33. $p<.001$. and a quadratic. $F(1,270)=4.54$, $p<.05$. component. For the spontaneous SCRs, the decrease comprised a linear trend. $F(1.270)=6.98$. $\mathrm{p}<.01$, and a tendency towards a quadratic one. $F(1.270)=3.03, p<.10$. For neither of the two variables was the effect of groups or Groups by Blocks significant.

\section{Interrelations Between Measures of Arousal and Habituation}

The interrelations of the following two sets of variables were computed over all 30 Ss with the Pearson correlation coefficient. Habituation: (1) time to habituation, (2) total number of specific SCRs. Arousal: (1) time to sleep onset. (2) total amount of weighted sleep, (3) number of spontaneous SCRs during the first

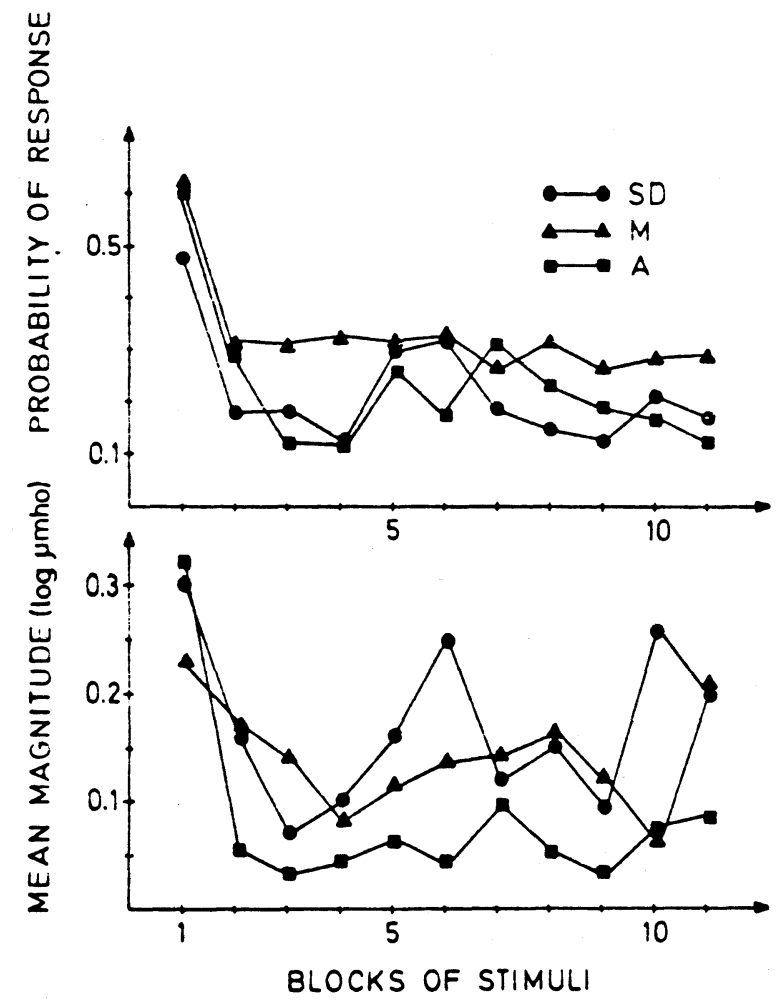

Fig. 2. Habituation of specific skin conductance responses for the sleep-deprived (SD), morning (M), and afternoon (A) groups over 11 stimulus blocks. The upper part depicts probability of response, whereas the lower part represents magnitude of response.

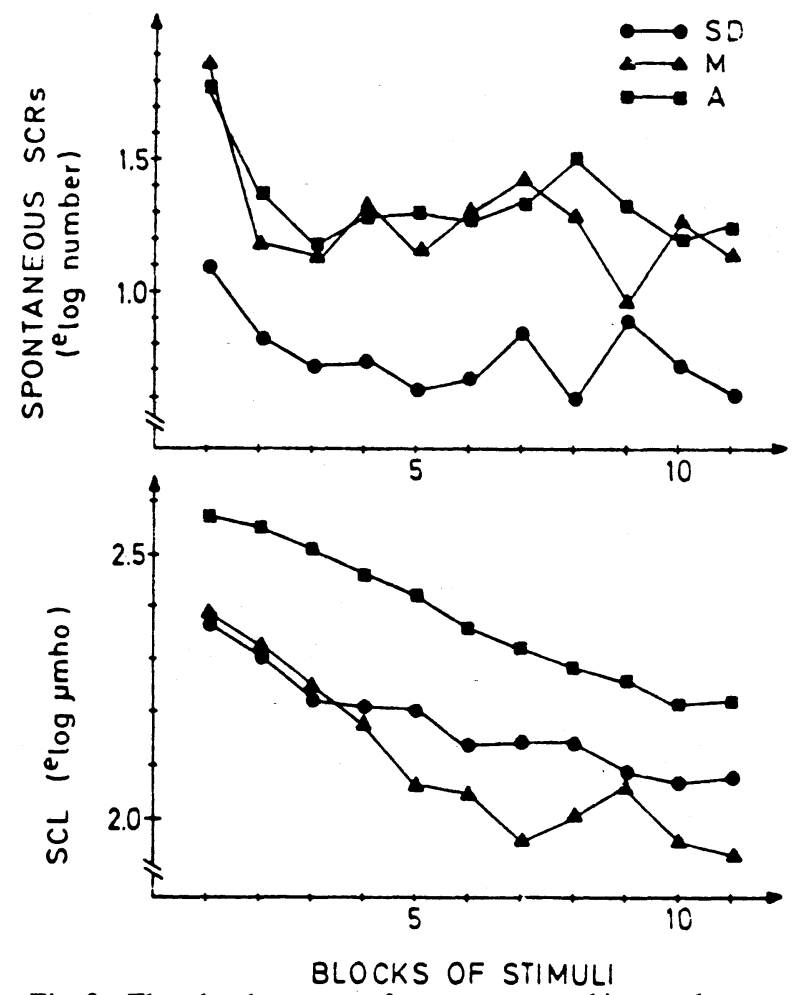

Fig. 3. The development of spontaneous skin conductance responses (upper part) and skin conductance level (lower part) over the 11 stimulus blocks for the sleep-deprived (SD), morning (M), and afternoon (A) groups.

stimulus biock (see Bohlin. in press b). As can be seen from Table 1, the two measures of habituation were significantly related to time to sleep onset and to initial number of spontaneous SCRs. In addition, the total number of specific SCRs showed a significant relationship with the total amount of weighted sleep.

\section{DISCUSSION}

The results demonstrated a faster occurrence of sleep. and more overall drowsiness and sleep, for the SD group compared to the other two groups, who did not differ

Table 1

The Intercorrelations Between Measures of Arousal and Habituation Over All 30 Sst

\begin{tabular}{ccc}
\hline & $\begin{array}{c}\text { Total } \\
\text { Time to } \\
\text { Habituation }\end{array}$ & $\begin{array}{c}\text { Number of } \\
\text { Specific } \\
\text { SCRs }\end{array}$ \\
\hline Time to Sleep Onset & $.352^{*}$ & $.584^{* *}$ \\
S & $(.347)$ & $(.439)$ \\
SD & $(.493)$ & $(.895)$ \\
Total Amount of Sleep & -.292 & $-.485^{* *}$ \\
S & $(-.447)$ & $(-.518)$ \\
SD & $(-.270)$ & $(-.575)$ \\
Initial Number of Spontaneous SCRs & $.701^{* *}$ & $.683^{* *}$ \\
S & $(.923)$ & $(.799)$ \\
SD & $(.415)$ & $(.824)$ \\
\hline
\end{tabular}

$\div$ The coefficients computed separately for the $S(N=20)$ and $S D(N=10)$ groups are given within parentheses.

${ }^{*} p<.05 .{ }^{* *} p<.01$ 
between themselves. Consonantly, there was a tendency, $F(2.27)=2.67, p<.10$, for the groups to differ in initial frequency of spontaneous SCRs (see Fig. 3), a variable previously shown to be a useful index of arousal differences (Bohlin, in press b). For all three groups, the SCL and spontaneous SCRs decreased over blocks, but the rate of decrease did not differ between them. In sum, the SD is considered to have been effective in bringing about a change in arousal level, whereas this was not true for the diurnal variation.

Concerning the relation between level of arousal and SCR habituation, the results present a complex picture. The low-arousal group (SD) did not differ from the other two groups in rate of habituation, irrespective of whether rate was measured as time to reach criterion or as slope of response function. On the other hand, measures of habituation were significantly correlated with indices of arousal, both spontaneous SCRs and sleep. Hence, an experimentally induced arousal decrease did not affect rate of habituation, although an intraindividual relationship between the two variables was obtained. An interpretation of these findings seems to necessitate a third construct. Thus, by hypothesizing a variable of individual differences, which exerts partial control over both arousal level and habituation, the contradictory results might be understood. This factor of individual differences would be seen to cause a covariation between arousal and habituation, empirically observed as a correlation between their indices. In addition, however, certain environmental factors might affect arousal or habituation directly, leading to a dissociation of effect on them.

The above interpretation casts new light on the previously presented data (Bohlin, 1973), which were taken to suggest an interactive relation between arousal and habituation. Since, in that study, arousal differences were indicated by individual differences in spontaneous SCRs, the apparent interaction might be referrable to the hypothesized dimension of individual differences; Ss at the high end of the dimension would be characterized by slow SCR habituation, high initial autonomic arousal, and little arousal decrease as effect of the habituation procedure, whereas Ss lower on the dimension would be more rapid to habituate, be lower in autonomic arousal, and, with respect to sleep tendency, be more affected by the habituation procedure. Furthermore. there is evidence for a wider generality of the proposed dimension, since electrodermal conditioning (Öhman \& Bohlin, 1973, in press), vigilance performance (Coles \& Gale, 1971; Siddle, 1962), and anxiety symptoms (Lader \& Wing, 1966) have also been shown to be related to either SCR habituation, spontaneous SCRs, or both.

Finally, the failure of finding an effect of lowered arousal on SCR habituation has some bearing upon considerations of the process of habituation. Thus, conceptions of response decrement in terms of two processes. a general, long-term one (arousal) as well as a specific, short-term one (Groves \& Thompson, 1970; Maltzman et a!, 1971) are not supported. Although it cannot be excluded that response decrement could be delayed by increased arousal (e.g., stress), the arousal decrease seen during repetitive stimulation does not seem to be an integral part of the observed habituation. If so, the increased rate of arousal decrease for the SD group should have been paralleled by higher rate of SCR habituation.

In conclusion, the present findings are taken as evidence of a differential effect of SD on arousal and SCR habituation. Concerning the relationship between the constructs behind the two latter variables, the picture of a one-way relationship emerges. Evidence lending credence to a dearousing effect of habituation has been previously presented (Bohlin, 1971), while from the present data, an effect of arousal on habituation appears unlikely. However, the data also attest to the previously documented (Johnson, 1963; Koepke \& Pribram, 1966; Katkin \& McCubbin, 1969; Lader \& Wing, 1966; Maltzman et al, 1971; Thayer \& Silber, 1971; Verbaten, 1972; Bohlin, 1972, 1973) relation between measures of arousal and habituation, suggesting an interpretation of it in terms of an underlying variable of individual differences, rather than as evidence for a causal relation.

\section{REFERENCES}

Bohlin, G. Monotonous stimulation, sleep onset, and habituation of the orienting reaction. Electroencephalography \& Clinical Neurophysiology, 1971, 31, 593-601.

Bohlin, G. Susceptibility to sleep during a habituation procedure as related to individual differences. Journal of Experimental Research in Personality, 1972, 6, 248-254.

Bohlin, G. Interaction of arousal and habituation in the development of sleep during monotonous stimulation. Biological Psychology, 1973, 1, in press.

Coles, M. G. H.. \& Gale, A. Physiological activity as a predictor of performance in a vigilance task. Psychophysiology, 1971, 8, 594-599.

Colquhoun, W. P. Circadian variations in mental efficiency. In W. $\mathrm{P}$. Colquhoun (Ed.), Biological rhythms and performance. London: Academic Press. 1971. Pp. 39-107.

Edelberg, R. Electrical properties of the skin. In C. C. Brown (Ed.), Methods in psychophysiology. Baltimore: Williams \& Wilkins, 1967. Pp. 1-53.

Groves. P. M.. \& Thompson. R. F. Habituation: A dual-process theory. Psychological Review, 1970, 77, 419-450.

Hinde, R. Behavioural habituation. In G. Horn and R. Hinde (Eds.), Short-term changes in neural activity and behaviour. Cambridge: Cambridge University Press, 1970.

Johnson, L. C. Some attributes of spontaneous autonomic activity. Journal of Comparative \& Physiological Psychology, 1963. 56, 415-422.

Johnson, L. C., \& Lubin. A. The orienting reflex during waking and sleeping. Electroencephalography \& Clinical Neurophysiology, 1967. 22. 11-21.

Katkin, E. S., \& IICCubbin. R. J. Habituation of the orienting response as a function of individual differences in anxiety and autonomic lability. Journal of Abnormal Psychology. 1969, 74. $54-60$.

Kirk. R. E. Experimental design: Procedures for the behavioral sciences. Belmont: Wadsworth. 1968. 
Koepke. J. E.. \& Pribram. K. H. Habituation of GSR as a function of stimulus duration and spontaneous activity. Journal of Comparative \& Physiological Psychology. 1966. 61. 442-448.

Lader. M. H.. \& Mathews. A. M. A phỵsiological model of phobic anxiety and desensitization. Behaviour Research \& Therapy. 1968. 6. 411-421.

Lader. M. H.. \& Wing. L. Physiological measures, sedative drugs and morbid anxietr. London: Oxford University Press. 1966.

Lynn. R. Attention. arousal and the orientation reaction. Oxford: Pergamon Press, 1966.

Maltzman. I.. Smith. M. J.. Kantor. W.. \& Mandell. M. P. Effects of stress on habituation of the orienting reflex. Journal of Experimental Psychology, 1971, 87, 207-214.

McDonald. D. G.. Johnson. L. C.. \& Hord, D. J. Habituation of the orienting response in alert and drowsy subjects. Psychophysiology, 1964, 1, 163-173.

Öhman. A.. \& Bohlin. G. Magnitude and habituation of the orienting reaction as predictors of discriminative electrodermal conditioning. Journal of Experimental Research in Personality, in press. a.

Öhman, A., \& Bohlin, G. Relation of spontaneous skin conductance fluctuations to simple and discriminative electrodermal conditioning. Journal of Experimental Research in Personality, 1973, 6, 293-299.

Öhman. A., \& Bohlin, G. The relationship between spontaneous and stimulus-correlated electrodermal responses in simple and discriminative conditioning paradigms. Psychophysiology, in press.
Oswald. I. Sleeping and wahing. Amsterdam: Elsevier. 1962.

Scholander. T. Habituation of autonomic response elements under two conditions of alertness. Acta Physiologica Scandinavica. 1960. 50. 259-268.

Scholander. T. The effects of moderate sleep deprivation on the habituation of autonomic response elements. Acta Physiologica Scandinavica, 1961, 51, 325-342.

Siddle, D. A. Vigilance decrement and speed of habituation of the GSR component of the orienting response. British Journal of Psychology, 1972.63. 191-194.

Sokolov, E. N. Perception and the conditioned reflex. New York: Pergamon Press, 1963.

Thayer. J.. \& Silber, D. E. Relationship between levels of arousal and responsiveness among schizophrenic and normal subjects. Journal of Abnormal Psychology, 1971, 77. 162-173.

Tizard, B. Evoked changes in EEG and electrodermal activity. during the waking and sleeping states. Electroencephalography \& Clinical Neurophysiology, 1966, 20, 122-128.

Verbaten. M. N. The influence of subject variables on rate of habituation of the orienting reflex. Psychological Laboratory. University of Ltrecht, Netherlands. Report No. 4. 1972.

(Received for publication February 6, 1973: revision received June 15. 1973: accepted June 18. 1973.) 